\title{
OPEN Regulation of adult neurogenesis by the endocannabinoid-producing enzyme diacylglycerol lipase alpha (DAGLa)
}

\author{
Lena-Louise Schuele, Britta Schuermann, Andras Bilkei-Gorzo, Sara Gorgzadeh, \\ Andreas Zimmer ${ }^{\mathbb{D}}{ }^{\text {\& Este Leidmaa }}$
}

The endocannabinoid system modulates adult hippocampal neurogenesis by promoting the proliferation and survival of neural stem and progenitor cells (NSPCs). This is demonstrated by the disruption of adult neurogenesis under two experimental conditions: (1) NSPC-specific deletion of cannabinoid receptors and (2) constitutive deletion of the enzyme diacylglycerol lipase alpha (DAGLa) which produces the endocannabinoid 2-arachidonoylglycerol (2-AG). However, the specific cell types producing 2-AG relevant to neurogenesis remain unknown. Here we sought to identify the cellular source of endocannabinoids in the subgranular zone of the dentate gyrus (DG) in hippocampus, an important neurogenic niche. For this purpose, we used two complementary Cre-deleter mouse strains to delete Dagla either in neurons, or in astroglia and NSPCs. Surprisingly, neurogenesis was not altered in mice bearing a deletion of Dagla in neurons (Syn-Dagla KO), although neurons are the main source for the endocannabinoids in the brain. In contrast, a specific inducible deletion of Dagla in NPSCs and astrocytes (GLAST-CreERT2-Dagla KO) resulted in a strongly impaired neurogenesis with a $50 \%$ decrease in proliferation of newborn cells. These results identify Dagla in NSPCs in the DG or in astrocytes as a prominent regulator of adult hippocampal neurogenesis. We also show a reduction of Daglb expression in GLAST-CreERT2-Dagla KO mice, which may have contributed to the neurogenesis phenotype.

Adult neurogenesis is a process in which new neurons are continuously generated in the adult mammalian brain $^{1}$. In particular, two major regions containing neural stem and progenitor cells (NSPCs) were identified as neurogenic niches, the subgranular zone (SGZ) of hippocampal dentate gyrus (DG) and the subventricular zone (SVZ). Even though the biological role of adult neurogenesis is far from being fully elucidated, it is now well established that new adult-born neurons from SVZ and SGZ are functionally incorporated in the olfactory bulb or hippocampus, respectively, participating in the function of this areas ${ }^{2}$. It is also evident that neurogenesis declines with aging in animals and possibly in humans ${ }^{3-5}$, particularly in the context of aging-related neurodegenerative disease ${ }^{6}$.

Adult neurogenesis is regulated by a variety of different intrinsic and extrinsic factors. Several studies revealed that endocannabinoid signaling plays a crucial role in the regulation of proliferation, differentiation and survival of progenitor cells in the neurogenic niches. Specifically, cannabinoid receptor 1 (CB1) KO mice, lacking the main endogenous cannabinoid receptor of the CNS, were found to show highly decreased adult neurogenesis ${ }^{7}$. Similarly, diacylglycerol lipase alpha (Dagla) deficient mice, lacking the main producing enzyme of the most abundant cannabinoid 2-arachidonoylglycerol (2-AG) displayed impairments in adult neurogenesis ${ }^{8,9}$. Interestingly, the activity of the endocannabinoid system seems to be also reduced in aged brains ${ }^{10,11}$. However, the cell type that regulates adult neurogenesis by endocannabinoids is not yet identified. Regarding CB1 signaling, a very recent study found that deleting CB1 specifically in NSPCs using an inducible Nestin-CreERT2 strain was sufficient to lead to reduced proliferation of progenitors in the DG of adult mice. The reduction of adult neurogenesis in this mouse line led to a concomitant depressive-like behavior of the mice, as assessed in the forced swim test ${ }^{12}$. Nevertheless, it is unknown where the endocannabinoids that activate CB1 receptors on progenitor cells come 
A

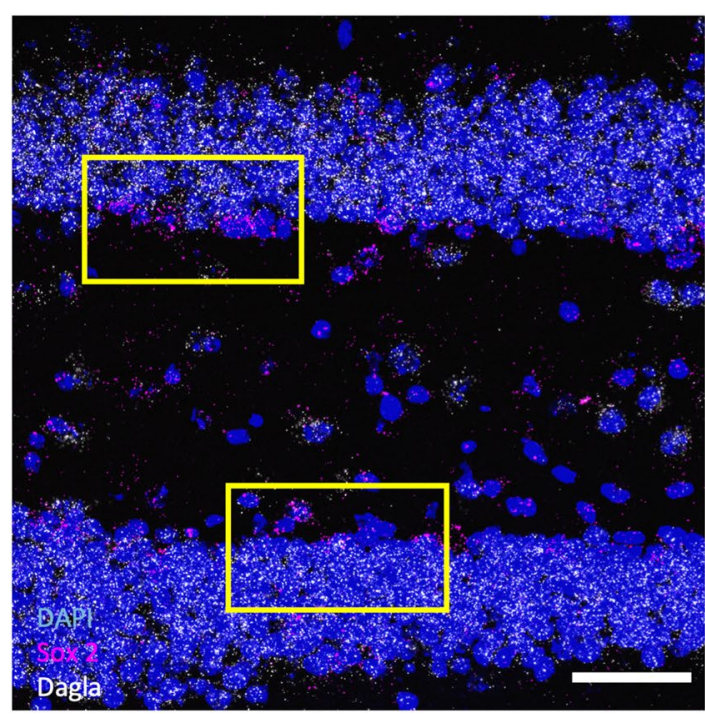

B

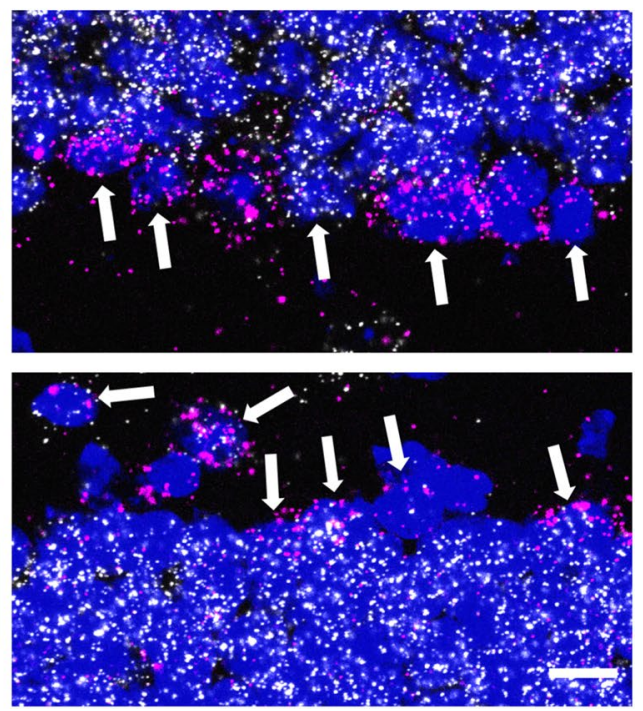

Figure 1. Dagla is expressed in neural stem and progenitor cells (NSPCs). (A) Representative image of an RNAscope in situ hybridisation assay detecting transcripts of diacylglycerol lipase alpha Dagla (white) and neural progenitor marker Sox 2 (magenta) in the dentate gyrus of hippocampus (DG) (scale bar $50 \mu \mathrm{m})$. (B) The magnified insets (scale bar $10 \mu \mathrm{m}$ ) show the subgranular zone (SGZ); the progenitor layer in the DG. White arrows indicate Sox 2-positive cells that co-express Dagla (scale bar $10 \mu \mathrm{m}$ ).

from. Elucidating this was the aim of this study. Therefore, conditional Dagla KO mice with a deletion in either neurons or astrocytes and NSPCs were used.

\section{Results}

Neural stem and progenitor cells (NPSCs) in subgranular zone (SGZ) of hippocampus express Dagla. Using a specific and sensitive in situ hybridisation method (RNAscope), we identified neural stem and progenitor cells (NSPCs) in the subgranular zone (SGZ) of the hippocampal dentate gyrus (DG) by the expression of Sox2. These cells also express Dagla (Fig. 1), although the expression level seems to be somewhat lower compared to the cells in the granular layer. To validate this result on the protein level, we also performed immunostainings with a DAGLa specific antibody. NSPCs were identified by their localization, morphology and GFAP expression. As shown in Fig. 3B, GFAP-positive cells with a morphology typical for NSPCs in the SGZ of control mice were also positive for DAGLa. Together, these results demonstrate that, in addition to neurons and astrocytes, NSPCs also express DAGLa.

Hippocampal deletion of DAGLa. The two conditional Cre-deleter strains used in this study (Syn-Cre and GLAST-CreERT2) showed very different but complementary Cre expression patterns in the hippocampal DG and cornu ammonis 3 (CA3) regions. Thus, as shown in Fig. 2A using RosaTomato reporter mice, Syn-Cre mice specifically expressed Cre in virtually all neurons of the DG and CA3. Please note that the expression of the RosaTomato reporter reveals those cells in which the Cre gene was active and thus Dagla will be deleted. In contrast, GLAST-CreERT2 mice showed no expression of tdTomato in neurons, but in astroglial cells (for quantification $s^{13}$ ). In the DG, cells expressing tdTomato were mainly NSPCs, because they were located in the neurogenic niche of the SGZ and showed the typical shape and GFAP expression of NSPCs (Fig. 2B left). Using a co-staining with the proliferation marker Ki67, we found that $68 \%$ of the proliferating cells in the DG expressed tdTomato (Fig. 2B right). This indicates that GLAST-CreERT2 is also active in NSCs and NPCs, confirming the findings previously reported by Mori et al., ${ }^{14}$. We therefore crossed Syn-Cre and GLAST-CreERT2 deleter strains with floxed Dagla (Dagla fl/fl) mice, thus deleting DAGLa in the corresponding cell populations. This was confirmed by immunohistochemisty (Fig. 2C,D), which showed a moderate reduction of the DAGLa signal in tamoxifen-induced GLAST-CreERT2-Dagla KO mice. In Syn-Dagla KO mice the immunostaining of DAGLa was markedly reduced in the CA1 region and virtually abolished in the DG and CA3 areas, consistent with a deletion of DAGLa in DG neurons. The quantification of DAGLa signal intensity in the DG showed a reduction of $76.4 \%$ for Syn-Dagla KO and $23.9 \%$ for GLAST-Dagla KO. These observations were further validated using RNAscope in situ hybridisation. Dagla expression was still present in the SGZ in Syn-Dagla mice despite the deletion in all other DG cells. An opposing picture was observed in GLAST-Dagla mice, where Dagla was reduced in the progenitor cell layer but not in the rest of the DG (Fig. 3A). A co-immunostaining with a marker for neurons (NeuN), and a marker for astrocytes and NSPCs (GFAP) showed that the same holds true on the protein level. DAGLa is expressed in GFAP-positive NSPCs in Syn-Dagla mice but not present in the neurons in granular cell layer, whereas the opposite is the case for GLAST-CreERT2-Dagla mice (Fig. 3B). Thus, in 
A

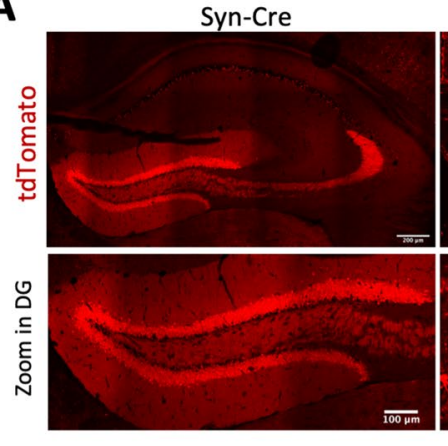

C

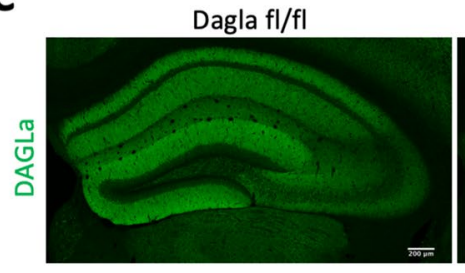

GLAST-CreERT2

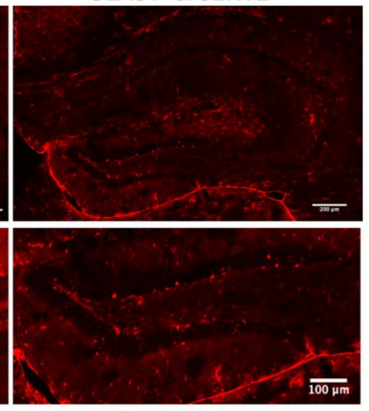

Syn-Dagla KO
B
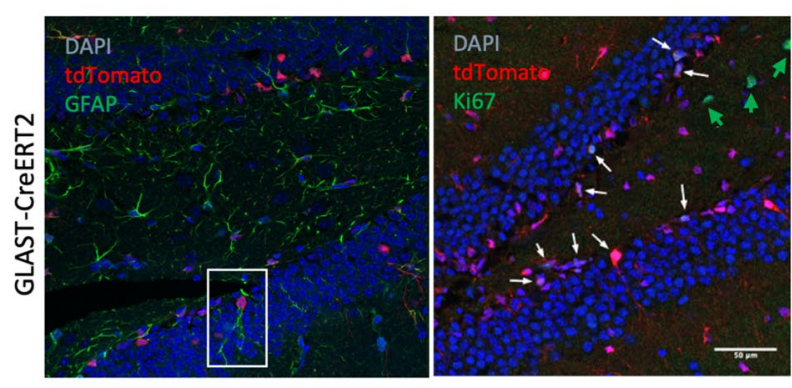

D

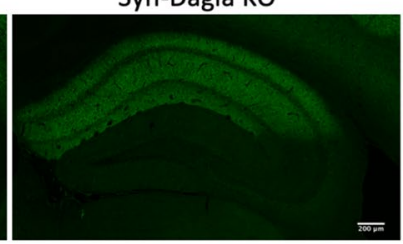

GLAST-CreERT2-Dagla KO

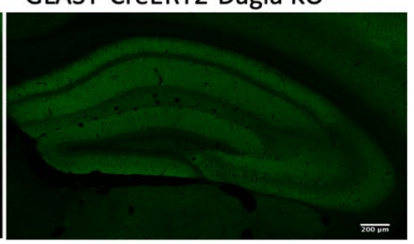

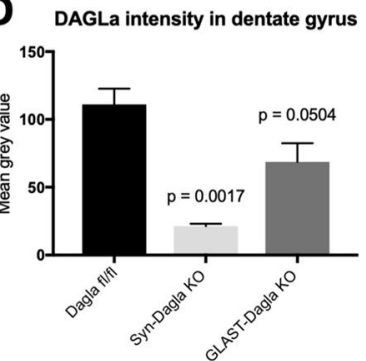

Figure 2. Syn-Dagla KO and GLAST-CreERT2-Dagla KO lines show opposing patterns of DAGLa deletion in the hippocampal dentate gyrus (DG). (A) Validation of Cre-efficacy in hippocampus (top, scale bar $200 \mu \mathrm{m}$ ) and DG (bottom, scale bar: $100 \mu \mathrm{m}$ ) in Syn-Cre and tamoxifen-induced GLAST-CreERT2 lines crossed with the RosaTomato reporter line. GLAST-CreERT2 show tdTomato expression (red) and thus recombination activity in areas populated mostly by glial cells, especially in the subgranular zone (SGZ) of DG, but not in neurons in the granule cell layer of DG. Syn-Cre mice show tdTomato expression specifically in neurons in the granule cell layer of DG and CA3 but not in CA1 and CA2 or SGZ. (B) (Left) Co-staining of GLAST-CreERT2-RosaTomato with glial marker GFAP (green) in dentate gyrus. TdTomato expressing glia cells are mainly localized in the subgranular zone (SGZ) of dentate gyrus and show typical shape of neural stem and progenitor cells (NSPCs, example in white box)). Only few astrocytes not located in SGZ show tdTomato expression. (Right) Co-staining of GLAST-CreERT2-RosaTomato with proliferation marker Ki67 (green). 68\% of all proliferating cells express Cre; $17 \%$ of all Cre-expressing cells are proliferating cells $(\mathrm{n}=3$ animals/group; white arrows: cells co-expressing tdTomato and Ki67; green arrow: proliferating cells not expressing tdTomato). (C) Representative images of DAGLa immunostainings from hippocampi. In Dagla fl/fl control mice, DAGLa is highly expressed throughout the hippocampus. Syn-Dagla KO mice show a very low DAGLa signal in dentate gyrus (DG) and cornu ammonis 3 (CA3), while CA1 and CA2 show similar DAGLa expression as in Dagla fl/fl control mice. The DAGLa signal in hippocampus of GLAST-CreERT2-Dagla KO mice is markedly reduced compared to control mice. (D) Quantification of DAGLa signal intensity in the DG of conditional knockout mice. GLAST-CreERT2Dagla KO mice show a significant reduction of DAGLa signal in the dentate gyrus, that was even more pronounced in Syn-Dagla KO mice (1-way ANOVA: $\mathrm{F}_{2,6}=18.34 ; p=0.0028 ; \mathrm{n}=3$ per group).

Syn-Dagla mice Dagla was deleted from all DG cells, with the exception of NSPCs and few astrocytes, whereas, conversely, in GLAST-CreERT2-Dagla mice Dagla was deleted only in NSPCs and a few astrocytes.

Adult hippocampal neurogenesis in conditional Dagla KO mice. GLAST-Dagla KO mice showed a strongly reduced number of BrdU positive cells in the DG one day after the last BrdU injection, suggesting decreased proliferation of NSPCs in this mouse line (Fig. 4A,B). Also, 21 days after the last BrdU injection that is used to estimate the survival of newly born cells, GLAST-Dagla KO mice showed less BrdU positive cells compared to littermate control mice. Please note that the ratio of BrdU positive cells on days 1 and 21 was similar in both genotypes, suggesting that the survival of NSPCs was not affected per se. Differentiation, measured by co-localization of BrdU with either a neuronal marker (NeuN) or astrocytic markers (GFAP or S100ß) was also unchanged (Fig. 4C). Surprisingly, Syn-Dagla KO mice, with a complete deletion of Dagla in all DG neurons, showed neither changes in the proliferation nor in the survival of NSPC in the DG. As shown in Fig. 5, the number of BrdU positive cells was similar to that observed in littermate control mice on day 1 and day 21 after BrdU injections. Analysis of co-localization of BrdU with cell specific markers also showed that the differentiation of neural progenitors was not changed in Syn-Dagla KO mice (Fig. 5C).

In addition, we analyzed a third mouse-line, namely GFAP-Dagla KO mice (Supplementary Fig. 1A-C). These animals show a constitutive deletion of Dagla in NSPCs, astrocytes and neurons in the DG (Supplementary Fig. 1D), corresponding to the known expression pattern of GFAP. From its time-line, this deletion is comparable to the constitutive deletion of Dagla in Syn-Dagla KO that is also developmental, with the difference that in Syn-Dagla KO the deletion is in neurons whereas in GFAP-Dagla KO, Dagla is also deleted in NSPCs and astrocytes in the DG. Notably, the proliferation of NSPCs is reduced in adult GFAP-Dagla KO mice (Supplementary 
A

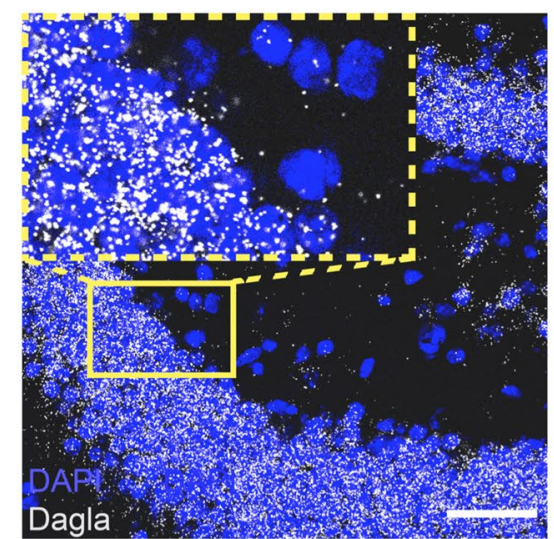

Syn-Dagla KO

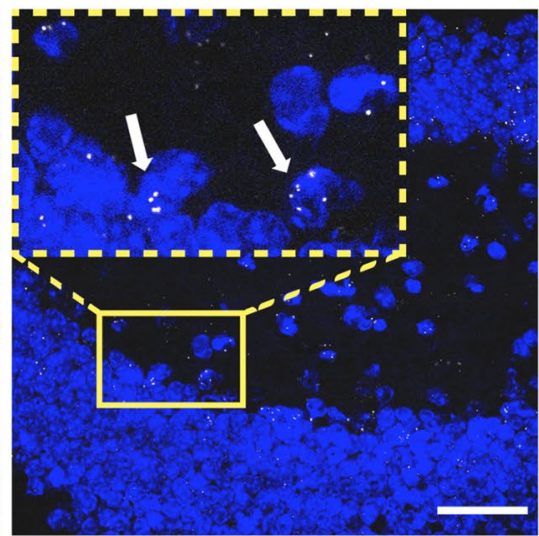

GLAST-ERT2-Dagla KO

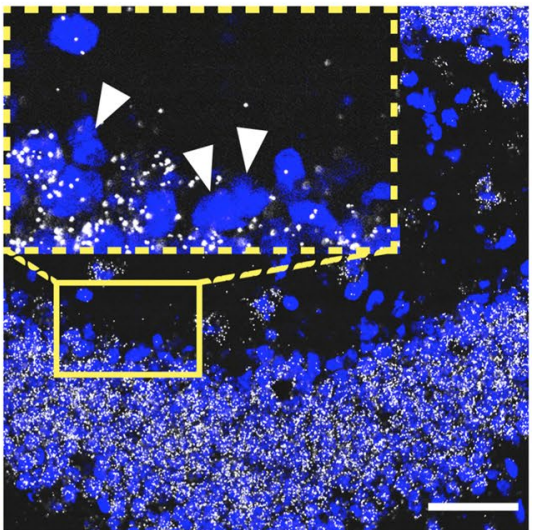

B
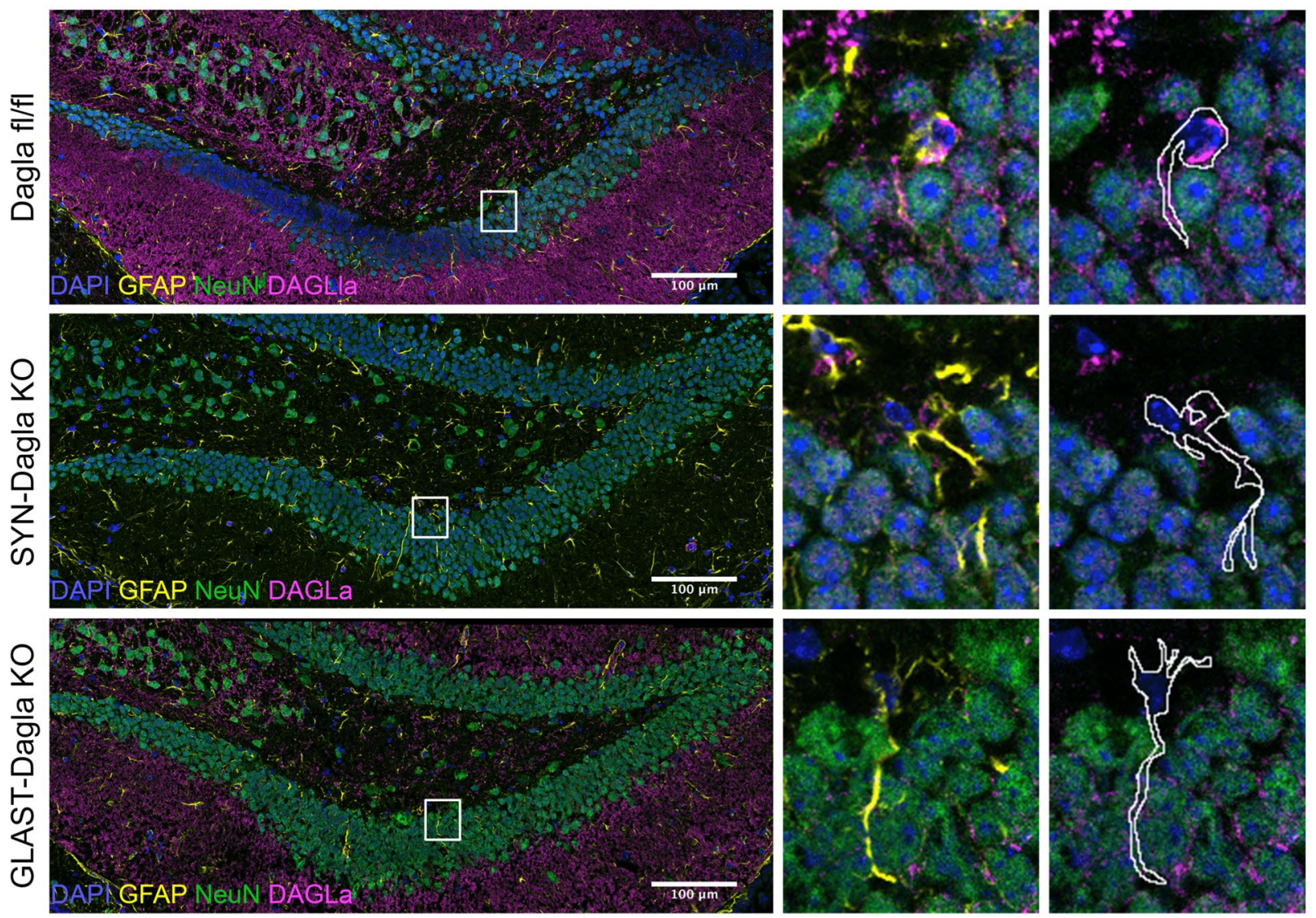

Figure 3. Dagla is deleted in the progenitor-containing subgranular zone (SGZ) in GLAST-CreERT2-Dagla KO but not in Syn-Dagla KO mice. (A) Representative images of an RNAscope in situ hybridisation assay detecting transcripts of Dagla (white) in the DG in the hippocampus of Dagla fl/fl control, Syn-Dagla KO and GLAST-CreERT2-Dagla KO mice. Dagla is deleted in the neuronal granular cell layer in Syn-Dagla and in the progenitor-containing subgranular zone (SGZ) in GLAST-CreERT2-Dagla KO mice (scale bar $50 \mu \mathrm{m}$ ). The magnified insets (scale bar $10 \mu \mathrm{m}$ ) show SGZ with white arrows indicating Dagla expressing cells in Syn-Dagla $\mathrm{KO}$ and white arrowheads Dagla-negative cells in GLAST-CreERT2-Dagla KO mice in the NSC layer ( $\mathrm{n}=3-4$ animals per group). (B) Immunostaining detecting DAGLa (magenta) co-localization with GFAP (yellow) and NeuN (green). The magnified insets show examples of GFAP-positive cells with NSPC morphology delineated with white. Progenitors express DAGLa in Dagla fl/fl control and Syn-Dagla KO SGZ and but not in GLASTCreERT2-Dagla KO. NeuN positive neurons in granule cell layer express DAGLa in control and GLASTCreERT2-Dagla KO sections, but not in Syn-Dagla KO (scale bar $10 \mu \mathrm{m}$ ). 
A
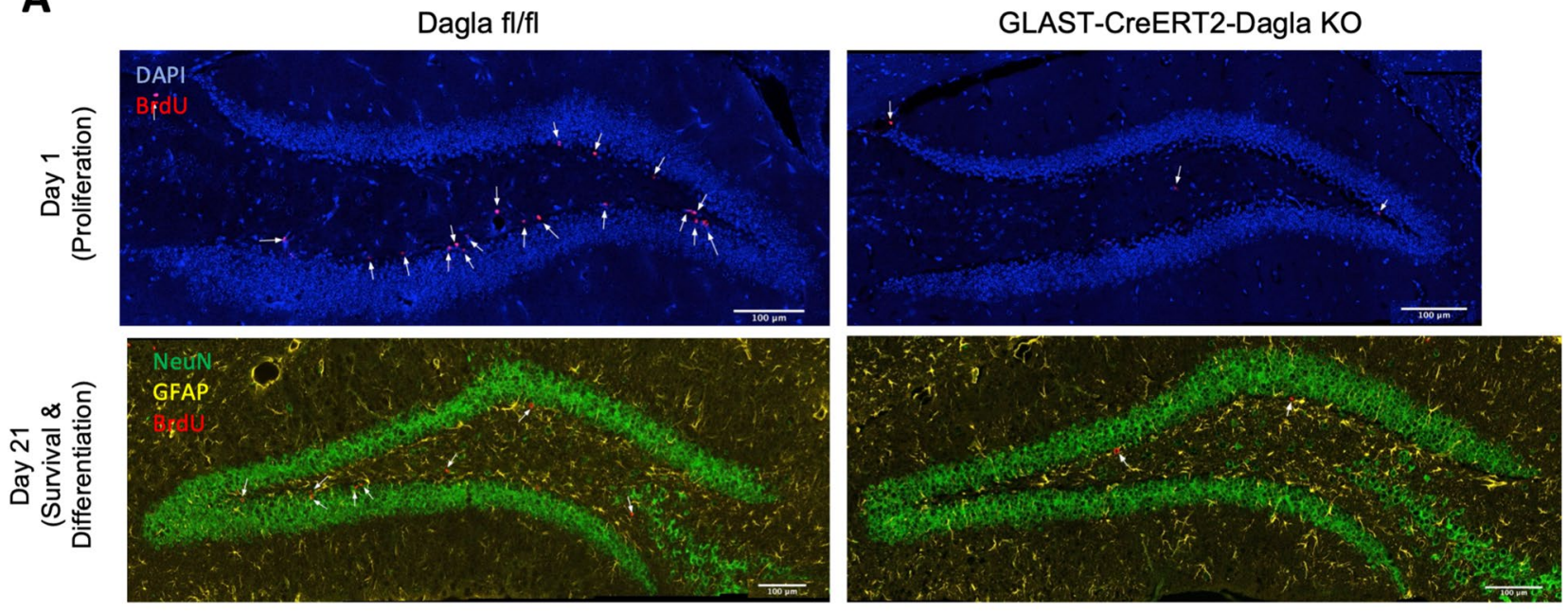

B

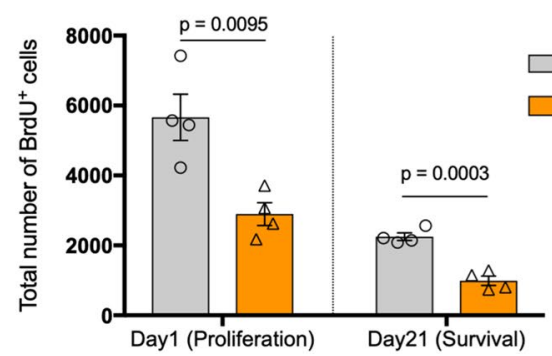

Dagla fl/fl

GLAST-Dagla KO
C

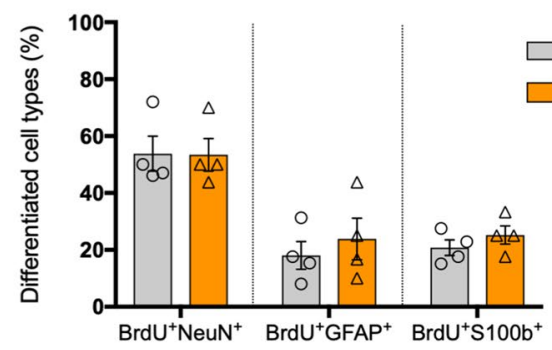

Dagla fl/fl

GLAST-Dagla KO

Figure 4. Adult hippocampal neurogenesis is impaired in GLAST-CreERT2-Dagla KO mice. (A) Representative immunohistochemistry micrographs of GLAST-CreERT2-Dagla WT and KO mice one or 21 days after BrdU injections in dentate gyrus to study proliferation and survival/differentiation, respectively (blue: DAPI; red: BrdU; green: NeuN; yellow: GFAP; scale bar: $100 \mu \mathrm{m}$ ). (B) The number of BrdU-positive cells in dentate gyrus of GLAST-CreERT2-Dagla KO mice was significantly lower compared to WT controls one day $(p=0.0095)$ as well as 21 days $(p=0.0003)$ after BrdU injections. (C) To analyze differentiation of progenitor cells, co-expression of BrdU-positive cells with neuronal marker (NeuN) or astrocytic marker (GFAP and S100) on day 21 were quantified. There were no changes in differentiation between GLAST-CreERT2-Dagla KO and WT control mice. Values represent mean \pm SEM; $n=4$ animals/group; 6 analyzed pictures per animal. Student's t-test.

Fig. 1B,C) in a very similar manner to the GLAST-CreERT2-Dagla KO mice (Fig. 4B,C) that have the Dagla deletion induced only in adulthood. Hence, it can be concluded that the deletion of Dagla in NSPCs and astrocytes either during development or in adulthood leads to a similar neurogenic phenotype.

Characterisation of Daglb expression in conditional Dagla KO mice. The DAGL protein has two isoforms, DAGLa and DAGLb. DAGLb is mostly studied in relation to inflammatory processes, but is also involved in adult neurogenesis. Since total knockout of Daglb also leads to impaired neurogenesis in the DG similar to that of Dagla ${ }^{8}$, we characterized the Daglb expression profile in the DG of GLAST-CreERT2-Dagla KO, Syn-Dagla KO, and total Dagla KO mouse lines (Fig. 6). Daglb was highly expressed in the granule cell layer of the DG, which mostly consists of neurons (Fig. 6A, cf Fig. 1A). Surprisingly, we found an approximately $60 \%$ reduction of Daglb expression in the hippocampal DG area in GLAST-CreERT2-Dagla KO and a lesser but also significant reduction of Daglb in Syn-Dagla KO mice (Fig. 6E). The significant decrease of Daglb in GLASTCreERT2-Dagla KO persisted when only the granule cells layer of DG region consisting mostly of neurons (excluding the hilus/polymorph layer of DG, and the molecular layer of DG) was quantified (Fig. 6D).

\section{Discussion}

In this study we show that the endocannabinoid 2-AG producing enzyme DAGLa is expressed in neural stem and progenitor cells (NSPCs) of the hippocampal dentate gyrus (DG) region, albeit at lower levels compared to neurons in the granule cell layer. Surprisingly, deleting DAGLa from NSPCs was important for the regulation of adult hippocampal neurogenesis, whereas deletion of neuronal Dagla did not affect neurogenesis. Specifically, we found that deleting Dagla from NSPCs in the DG using inducible GLAST-CreERT2-Dagla KO mice resulted in a prominent deficit in the proliferation of newborn cells.

We have recently quantified Dagla expression in many brain regions in wild type mice, as well as in the GLAST-CreERT2-Dagla KO strain used here ${ }^{13}$. Using the highly sensitive RNAscope method for in situ 
A

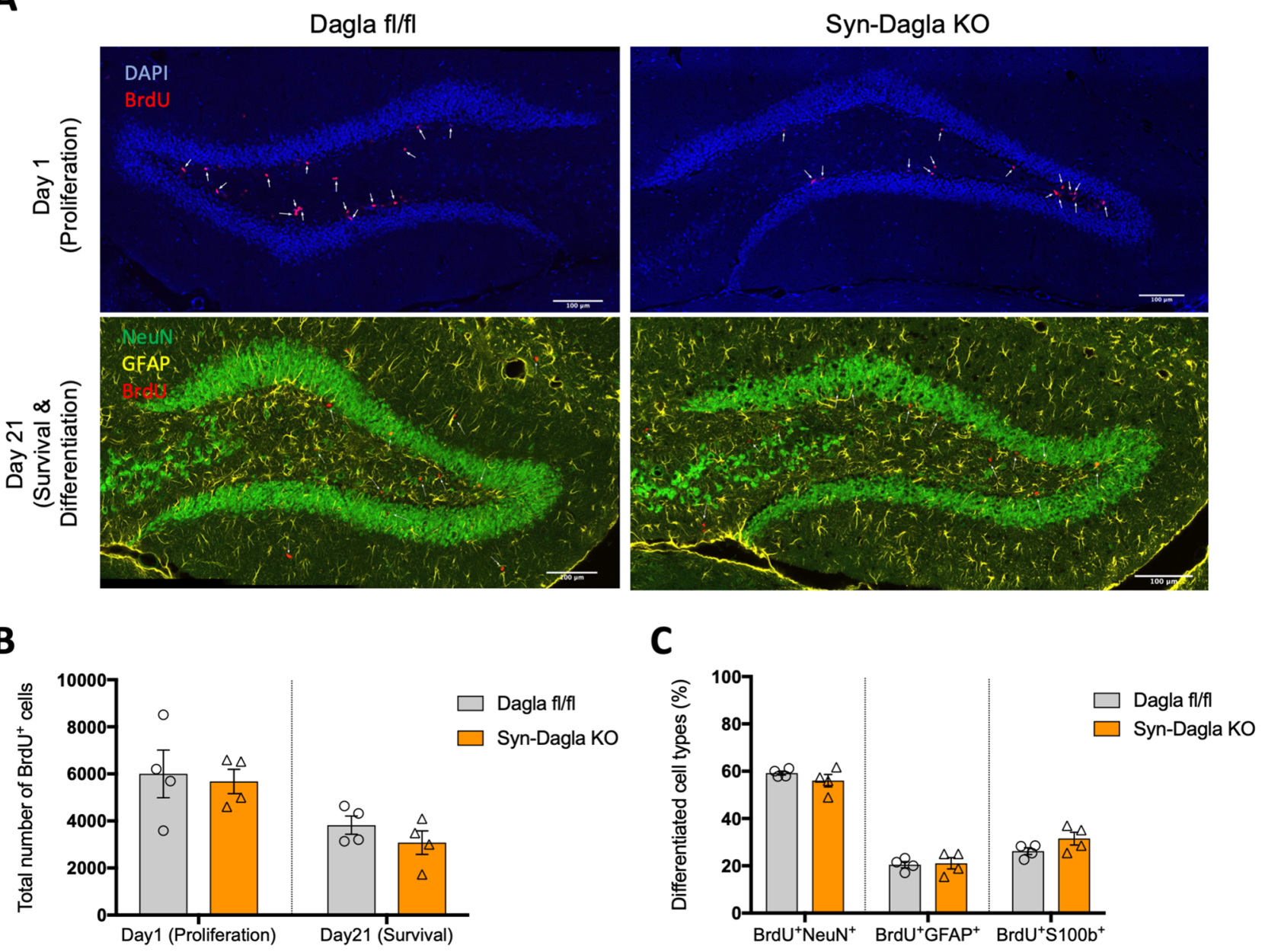

Figure 5. Adult hippocampal neurogenesis is not altered in Syn-Dagla KO mice. (A) Representative immunohistochemistry stainings of Syn-Dagla KO and control mice. Syn-Dagla KO mice show similar number of BrdU-positive cells (red) 1 day after the last injection. 21 days after BrdU injections, the number of BrdU positive cells does not differ between Syn-Dagla KO mice and controls. Additionally, brains were stained with an astrocytic marker GFAP (yellow) and a neuronal marker NeuN (green) to investigate differentiation, which was unchanged (scale bar: $100 \mu \mathrm{m}$ ). (B) The number of BrdU-positive cells in dentate gyrus of Syn-Dagla KO mice was similar to controls, one and 21 days after BrdU injections. (C) Differentiation of progenitor cells in dentate gyrus of Syn-Dagla KO mice. BrdU-positive cells were analyzed for co-expression of neuronal marker NeuN and astrocytic markers GFAP and S100beta. Values represent mean $\pm S E M ; n=4$ animals/group, 6 analyzed pictures/ animal. Student's t-test.

hybridization we previously demonstrated a specific deletion of Dagla in the GLAST-CreERT2-Dagla KO strain in astrocytes but not in neurons, in almost all brain regions. An exception was the hilus region of the DG where no Dagla deletion in astrocytes was found in this mouse line ${ }^{13}$. Thus, in the GLAST-CreERT2-Dagla KO strain Dagla was normally expressed in DG neurons, as well as astrocytes in the hilus. Our further analysis in the present paper demonstrated that Dagla was nevertheless deleted in the progenitor cell layer (NSPCs) of the DG in GLAST-CreERT2-Dagla KO mice. The second mouse line used in the current study, Syn-Dagla KO mice, showed a complementary pattern: a complete deletion of Dagla from all neurons in the DG, but an intact Dagla expression in NSPCs. These fortuitous opposing patterns enabled us to precisely interrogate the cellular source of $2-A G$ in the regulation of adult hippocampal neurogenesis.

The fact that hippocampal neurogenesis was completely normal in Syn-Dagla KO mice was striking and contrary to our expectations. Dagla is very highly expressed in the granule neurons in the DG region in control mice, whereas in the Syn-Dagla KO mice Dagla is drastically reduced in the DG area (see Figs. 2 and 3), almost to the level of constitutive Dagla KO mice ${ }^{13}$. Since constitutive Dagla KO mice do show impaired neurogenesis ${ }^{8}$, the expectation was the same for Syn-Dagla KO animals. Because the second isoform of Dagl, Daglb, has also been shown to regulate neurogenesis, and the lack of it reduces relative levels of cell proliferation in the SGZ, as does the lack of Dagla ${ }^{8}$, we also quantified Daglb expression in our mouse lines. In contrast to what has previously been published, we observed a prominent expression of Daglb (comparable to that of Dagla) in adult hippocampal neurons ${ }^{15}$. Furthermore, deleting Dagla from NSPCs resulted in a reduction of Daglb expression in the DG granule cell layer, whereas the deletion of Dagla from the neurons of DG had a smaller effect on Daglb 
A

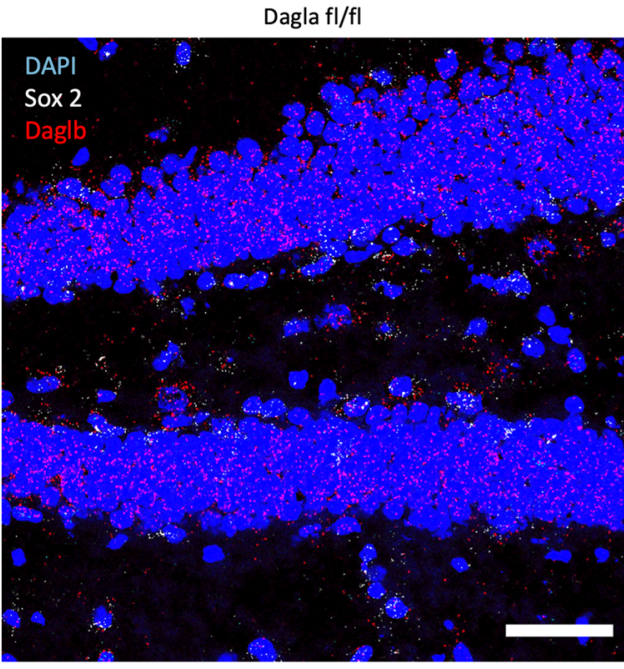

C

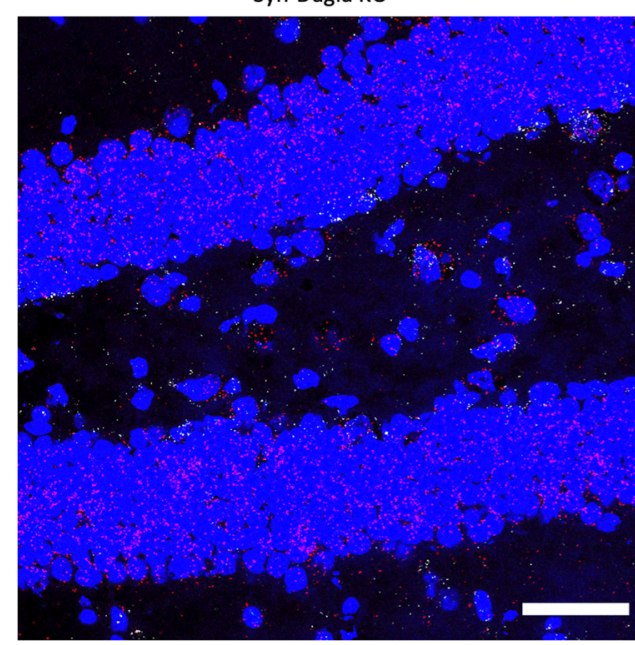

E

Hippocampal dentate gyrus, the whole image

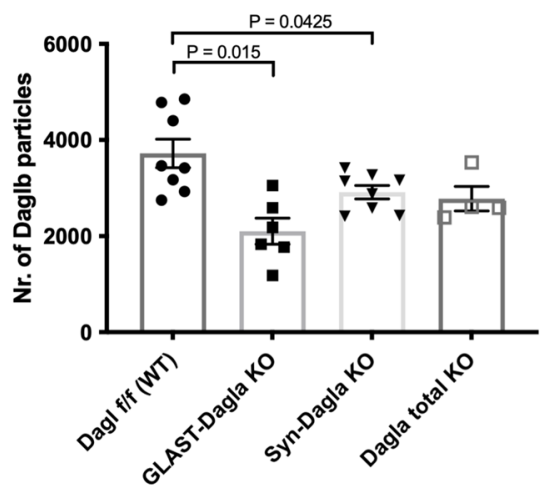

B

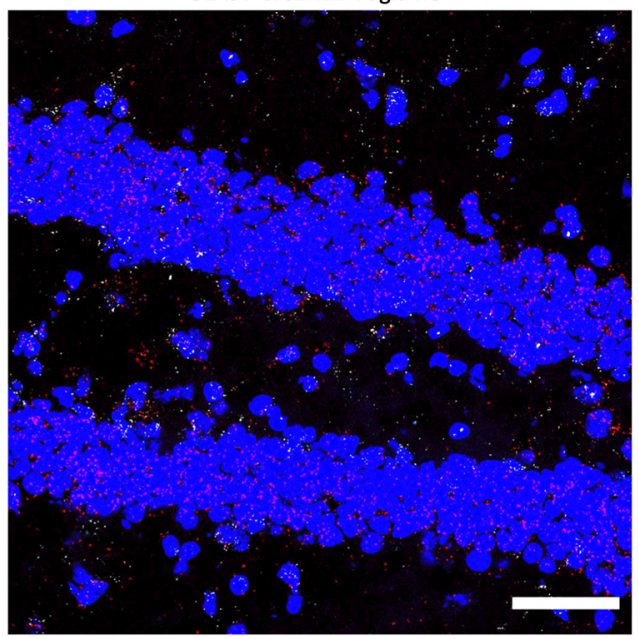

D

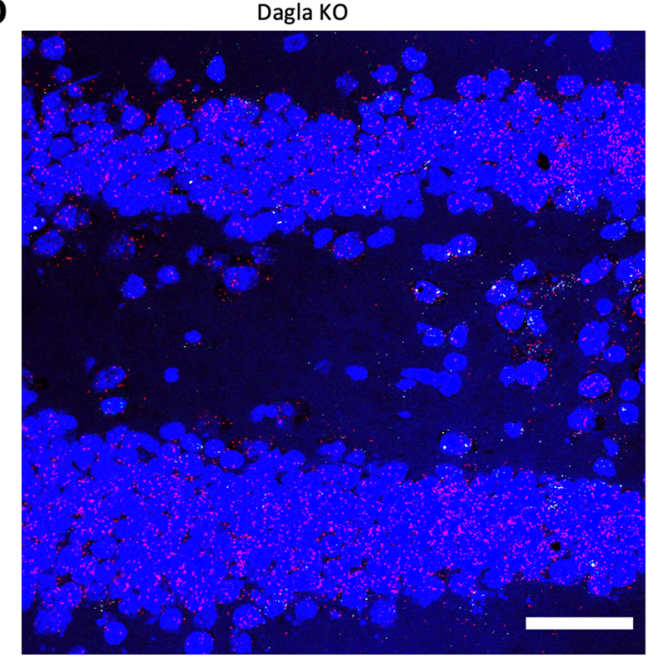

$\mathbf{F}$

Dentate gyrus, granule cell layer

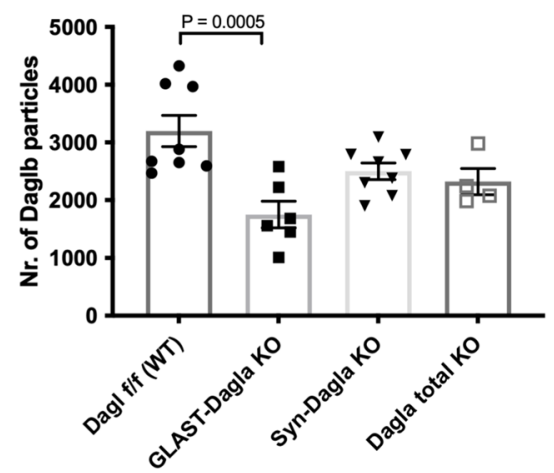

Figure 6. Daglb expression is downregulated in GLAST-CreERT2-Dagla KO mice. Representative $40 \times$ images of an RNAscope in situ hybridisation assay detecting transcripts of diacylglycerol lipase beta Daglb (red) and neural progenitor marker Sox 2 (white) in the dentate gyrus of hippocampus (DG) (scale bar $50 \mu \mathrm{m})$ (A) in Dagla fl/fl control mice, (B) in GLAST-CreERT2-Dagla KO with a deletion in astrocytes and neuronal progenitor cells, $(\mathbf{C})$ in constitutive neuronal Syn-Dagla KO animals, and (D) in total Dagla KO mice. Quantification of Daglb particle count at Bregma - $1.70 \mathrm{~mm}(\mathbf{E})$ in the whole hippocampal DG region (including the granule cell layer, the hilus/polymorph layer of DG, and the molecular layer of DG) $\left(\mathrm{F}_{3,22}=7.522\right.$, $p=0.0012)$ and $(\mathbf{F})$ only in the granule cell layer that consists mostly of neurons $\left(\mathrm{F}_{3,22}=7.256, p=0.0015\right)$. Values represent mean \pm SEM; $\mathrm{n}=2-4$ animals/group; 2 pictures analyzed per animal. 1-way ANOVA with Dunnett's multiple comparisons test. 
expression. This observation was at odds with a possibility that Daglb expression could have been increased to compensate for the deletion of Dagla and thus could have explained why Syn-Dagla KO mice still showed intact neurogenesis. Be that as it may, it is possible that the reduced levels of neuronal Daglb contributed to the reduced cell proliferation in the SGZ of GLAST-CreERT2-Dagla KO mice. The cellular and molecular mechanisms linking the expression of Dagla and Daglb in these different cell populations will be interesting to determine.

It is well-known that adult neurogenesis is influenced by physical activity, environmental factors and emotional states. Thus, environmental enrichment or running wheel activity can stimulate the proliferation and/or survival of NSPCs, whereas stress and ageing reduce progenitor cell proliferation ${ }^{16-18}$. Many studies have demonstrated that these factors modulate not only adult neurogenesis, but also affect cognitive functions, providing a link between adult neurogenesis and cognition and behavior. The mechanisms of how these environmental stimuli affect neurogenesis are not yet completely understood ${ }^{16}$, but seem to involve the neuronal activity-driven release of growth factors such as BDNF and FGF2. Considering the prominent role of the endocannabinoid system in the modulation of neuronal activity, we assumed that endocannabinoids produced by neurons as a result of neuronal activity would indirectly modulate NSPC proliferation and survival ${ }^{19,20}$. Contrary to this hypothesis, our present results could also support the possibility that endocannabinoids modulating NSPC proliferation could also originate from progenitors themselves ${ }^{21,22}$. Our findings are consistent with a recent report showing that cannabinoid receptor 1 (CB1) in NSPCs is also important for NSPC proliferation ${ }^{12}$. In this study CB1 was specifically deleted in NSPCs using a Nestin-CreERT2 deleter strain, whereas CB1 expression in DG neurons remained intact. These mice also showed a decreased proliferation of NSPCs ${ }^{12}$. Therefore, deleting the CB1 receptor in NSPCs produced a very similar phenotype as deleting the main ligand-producing enzyme DAGLa in NSPCs. The cellular fate of newly born cells in hippocampus was not altered in either GLAST-CreERT2-Dagla or Syn-Dagla KO mice. Specifically, the proportion of NSPCs that differentiated into NeuN-positive neurons or S100beta-positive astrocytes was not changed. Please note that the genotype-dependent reduction of BrdUpositive cells remained constant throughout the entire observation period ( $-50 \%$ in GLAST-CreERT2-Dagla KO mice on both days 1 and 21) and that most BrdU-positive cells also expressed markers for neurons or astrocytes on day 21 . This excludes the possibility that newborn cells simply failed to differentiate. Correspondingly, deleting CB1 in NSPCs in a previous study also resulted in the reduction of the number of both newly differentiated NeuN-positive neurons and S100beta-positive astrocytes ${ }^{12}$. Thus, the general profile of neurogenesis in GLASTCreERT2-Dagla KO mice resembled closely to that of CB1 KO in NSPCs. Together, these findings indicate that an endocannabinoid-mediated autocrine signaling mechanism in NSPCs may modulate adult hippocampal neurogenesis.

A similar autocrine mechanism has also been suggested during the development of the nervous system. DAGLa and CB1 are both expressed in migratory neuroblasts throughout the cell, including the tip of the filopodia in the terminal growth cone-like structure ${ }^{23}$. There is accumulating evidence to suggest that this autocrine mechanism contributes to the regulation of axonal growth and guidance, probably by acting downstream of a cell adhesion molecules/fibroblast growth factor receptor (CAM/FGFR) signaling system ${ }^{23}$. Specifically, FGFR activation induces $2-A G$ production by sequential activation of phospholipase $\mathrm{C} \gamma$ (PLC $\gamma$ ) which produces diacylglycerol, that in turn produces 2-AG through DAGLa. This results in CB1 activation in neuroblasts and modulates cortical neuron specification, elongation and morphological differentiation during embryonal development ${ }^{21,24,25}$.

An autocrine manner of cannabinoid receptor activation by 2-AG also has been suggested to happen in the adult brain ${ }^{21,23}$. Oligodendrocyte precursors also produce $2-A G$ and express $\mathrm{CB} 1$ and $\mathrm{CB} 2$ receptors and basal tone of 2-AG and cannabinoid receptors maintains the precursor proliferation in culture ${ }^{26}$, again hinting towards a possible autocrine mechanism regulating proliferation. Of note, the agonists of CB2 also stimulate progenitor proliferation in the subventricular zone in vivo, particularly in older animals ${ }^{27}$. However, the deletion of CB2 did not affect adult hippocampal neurogenesis in baseline conditions ${ }^{28}$. Whether the effects of CB2 on neurogenesis could also be autocrine and if these effects are dependent on aging is still to be determined.

The field of stem cell biology has gained attention and made progress in the recent decades because it holds great promise to repair and rejuvenate different tissues in the adult organism. The endocannabinoid system is a possible target for understanding these processes as it regulates stem cell biology in the whole organism throughout its life-span ${ }^{25}$. In early embryogenesis, endocannabinoids influence embryonic and trophoblast stem cell differentiation and survival. Its effects continue in ectoderm-derived neural tissues and mesoderm-derived hematopoietic and mesenchymal stem cells, thus affecting blood cells, adipose tissue, bone, muscle and epithelia ${ }^{21,25}$. Considering this, it is not surprising that total Dagla or CB1 knockouts show reduced breeding efficiency via smaller litters and less offspring. It is remarkable, however, that mice lacking Dagla or CB1 do not have gross developmental deficits ${ }^{9}$. A plausible explanation is that there is a redundancy of pathways and compensation by other factors regulating these crucial developmental stages; for example, by 2-AG generated by DAGLb, by anadamide levels, other fatty acid aminohydrolase enzyme substrates or arachidonate lipids ${ }^{8,27,29}$. In adulthood, however, there seems to be less plasticity and potential for compensation. This becomes important in natural aging processes in which 2-AG levels decrease ${ }^{10}$ and there is a depletion of NSPC in the hippocampus possibly leading to cognitive deficits. In fact, a chronic low dose of $\Delta 9$-tetrahydrocannabinol can restore the age-related decline on cognitive functions in old mice ${ }^{30}$.

We previously demonstrated that GLAST-CreERT2-Dagla KO mice had a profound impairment of maternal behavior and a depressive-like behavioral phenotype ${ }^{13}$. Depressive-like behavioral phenotype are shown to be a result of interfering with adult hippocampal neurogenesis ${ }^{31}$. Conversely, many animal models of depression show impaired hippocampal neurogenesis ${ }^{32}$. It is thus possible that the behavioral phenotype of GLAST-CreERT2Dagla KO mice was at least partially due the impaired hippocampal neurogenesis. This idea is supported by a study of Zimmermann et al. (2018), which showed that mice lacking CB1 on NSPCs also have a depression-like phenotype and reduced adult hippocampal neurogenesis ${ }^{12}$. 
Together, we propose that progenitor cells produce 2-AG which binds to their own CB1 receptors and promotes adult neurogenesis. DAGLb seems to be co-regulated with DAGLa and may also modulate adult hippocampal neurogenesis. Impairments in this process might eventually lead to development of depressive-like behaviors or aging-related cognitive decline.

\section{Methods}

Animals. Dagla fl/fl (B6.cg(Dagla)tm 1 Zim $)^{9}$ male mice on a C57BL/6J genetic background (2-5 month old) were used in this study. These mice were crossed to mouse lines expressing Cre recombinase either under the control of a neuron specific synapsin (Syn) promoter, under the control of an astrocyte-specific glutamate transporter promoter (GLAST) or under the control of glial fibrillary acidic protein (GFAP) promoter. The synapsinCre (B6.Cg-Tg(Syn1cre)671Jxm/J) and GFAP-Cre (B6.Cg-Tg(Gfap-Cre)73.12Mvs/J) lines was obtained from The Jackson Laboratory. Of note, using the maternal GFAP-Cre in breeding led to a germline transmission of the knockout, contrary to what was described by The Jackson Laboratory, hence only male GFAP-Cre mice were used in our breedings. The glutamate transporter-Cre line (B6.Cg-Slc1a3tm1(cre/ERT2)Mgoe) was generated by Mori et al., 2006 ${ }^{14}$. This strain has an insertion of a tamoxifen-inducible form of Cre (CreERT2) in the locus of astrocyte-specific glutamate aspartate transporter (GLAST, Slcla3tm1). The resulting mouse lines are referred to as Syn-Dagla and GLAST-CreERT2-Dagla, respectively. Mice were always bred heterozygously for Cre. Littermates not expressing the Cre gene, designated as "Dagla fl/fl", were used as controls. No inclusion or exclusion criteria were set besides obtaining the desired number of animals per genotype. The floxed Dagla allele, the wild type allele, the knocked out Dagla allele, and the Cre locus were identified by polymerase chain reaction (PCR) using appropriate primers (Cre1_fwd CATTT GGGCC AGCTA AACAT, Cre2_fwd GCATT TCTGG GGATT GCTTA, Cre1_rev CCCGG CAAAA CAGGT AGTTA, Cre2_rev TGCAT GATCT CCGGT ATTGA, Dagla_fwd TAG CTT AGC CCC CAT GTG AC, Dalga WT rev GAG ATG GGT TCC ACC TCC TT, Dagla fl/KO_rev CGC AGC CCA AAA GAT ACA AT). To validate region specific Cre expression we used RosaTomato reporter mice (The Jackson Laboratory (B6.Cg-Gt(ROSA)26Sortm14(CAG-tdTomato)Hze/J). They carry a reporter construct in the ubiquitously expressed Gt(ROSA)26Sor locus. The reporter is expressed after Cremediated excision of a loxP-flanked STOP cassette. We used mice that were heterozygous for RosaTomato (wt/ ins) and Cre (wt/tg). Mice were housed under a $12 \mathrm{~h}$ light/dark cycle (lights on $9 \mathrm{am}-9 \mathrm{pm}$ ) with ad libitum access to food and water. All experiments were approved by the North Rhine-Westphalia State Environment Agency (LANUV, Landesamt für Natur, Umwelt und Verbraucherschutz, licence nr: AZ: 84-02.04.2017.A234) and the were performed in accordance with the relevant guidelines and regulations. The study was conducted in compliance with the ARRIVE guidelines.

RNAscope assay. The RNAscope method was used to evaluate the expression of Dagla in the cells of interest (www.acdbio.com/RNAScope). For this purpose, mice $(n=2-4$ per genotype) were killed by decapitation, brains were quickly removed, flash frozen in dry ice-cooled isopentane and stored at $-80{ }^{\circ} \mathrm{C}$. Brains were cryosectioned at a thickness of $10 \mu \mathrm{m}$ and mounted on SuperFrost Plus slides (Thermo Fisher, Schwerte, Germany). The exact Bregma coordinates were identified from every twelfth slide according to Paxinos Brain atlas ${ }^{33}$. RNAscope Multiplex Fluorescent Reagent kit was used according to manufacturer's instructions (Advanced Cell Diagnostics, Newark, CA). The probes for Dagla (cat.no 478821) and Daglb (cat.no 497801) mRNA were multiplexed with the probes detecting the neural stem cell marker Sox2 (cat. no 402041). Confocal images of sections were obtained using a Leica TSC SP8 with $40 \times$ magnification $(291 \mu \mathrm{m} 2$ area $)$ and analyzed using Fiji software (version 2.0.0-rc-69; NIH, Bethesda, MD, USA). Micrographs from at least two consecutive sections from an animal were analyzed per brain region (Bregma $-1.34--1.46 \mathrm{~mm}$ ). In every assay, sections from constitutive Dagla KO mice ${ }^{9}$ were used as controls for non-specific binding of the Dagla probe.

Immunohistochemistry (IHC). Mice were anesthetized with isoflurane and transcardially perfused with $20 \mathrm{~mL}$ of cooled PBS, followed by $60 \mathrm{~mL}$ of $4 \%$ formaldehyde. The brains were postfixed in $4 \%$ formaldehyde (only for co-stainings with tdTomato) for one hour, incubated in $20 \%$ sucrose/PBS for approximately 3 days, and frozen in dry ice-cooled isopentane. Tissues were stored at $-80{ }^{\circ} \mathrm{C}$ until further processing and cut in a cryostat with a chamber temperature of $-22^{\circ} \mathrm{C}$. Slices were collected on SuperFrost Plus adhesion slides (Thermo Fisher) and stored at $-20^{\circ} \mathrm{C}$. For DAGLa staining, brains were cut in $40 \mu \mathrm{m}$ thick free-floating sections (Bregma -0.94 to $-3.34 \mathrm{~mm})$. For immunostainings with cell type specific markers and tdTomato, brains were cut into $16 \mu \mathrm{m}$ slices (Bregma - 1.46 until - 2.46, every 8th section collected on a slide). For DAGLa co-staining with cell-specific markers, fresh frozen brains were cut in $10 \mu \mathrm{m}$ thick slices (Bregma - 1.34- - 1.46) and post-fixed for $20 \mathrm{~min}$ in $4 \%$ formalin in phosphate buffer before staining. After immunostaining, brain slices were briefly washed in $\mathrm{ddH}_{2} \mathrm{O}$ and embedded in DAPI Fluoromount-G(R) media (Southern Biotechnology Associates. Inc., Birmingham, AL). When co-staining of DAGLa with cell-specific markers was performed, a mounting media ProLong $^{\text {Ta }}$ Diamond Antifade with DAPI (ThermoFisher) was used. Fluorescence images were obtained with the Leica SP8 confocal microscope, with 20x (NA 0.07, pixel size: $0.001 \mathrm{~mm} \times 0.001 \mathrm{~mm}$ ) or $40 \mathrm{x}$ (NA 1.1, pixel size: $0.142 \mu \mathrm{m} \times 0.142 \mu \mathrm{m}$ ) objective lenses. Phosphate buffer (PB, $0.2 \mathrm{M}, \mathrm{pH} 7.6)$ was used as a diluent and wash solution for DAGLa staining (DGLa-Rb-Af380; Frontier Institute Co., Ltd., Hokkaido, Japan). Triton (0.3\%) was used for permeabilization (10 min). Non-specific antibody binding was blocked with $5 \%$ normal goat serum and $0.2 \%$ Triton ( $1 \mathrm{~h}$ at RT), DAGLa antibodies were incubated overnight at RT (1:400 in blocking solution). Secondary goat anti-rabbit Alexa-Fluor647 (Life Technologies, Darmstadt, Germany) was incubated for $2 \mathrm{~h}$ at RT (1:500 in blocking solution). For DAGLa co-stainings with neuronal or astrocyte markers guniea pig antiNeuN (1:1000 266004, Synaptic Systems) and chicken anti-GFAP (1:1000 ab4674, Abcam, Cambridge, GB) were used. As secondary antibodies, goat anti-chicken Alexa488, goat anti-guniea pig Alexa568 and goat anti-rabbit 
Alexa647 were used. For tdTomato IHC phosphate-buffered saline (PBS; $0.01 \mathrm{M}$, pH 7.4) served as a general diluent and wash solution. Sections were permeabilized by $0.5 \%$ TritonX100 in PBS for one hour. Unspecific binding was blocked by $3 \%$ BSA for two hours prior to primary antibody incubation $\left(24 \mathrm{~h}\right.$ at $\left.4{ }^{\circ} \mathrm{C}\right)$. Microglia were stained with rabbit anti-Ibal antibody (1:2000 in 0.3\% BSA/PBS; 019-19741, Wako, Richmond, VA), astrocytes with rabbit-anti-S100ß (1:2000 ab41548, Abcam) and chicken anti-GFAP (1:2000 ab4674 Abcam), neurons with rabbit anti-NeuN (1:500 ABN78C3, Merck, Millipore, Burlington, USA). Secondary antibodies were antirabbit Alexa-Fluor 488 (1:1000), donkey anti-rabbit Alexa-Fluor 647 (1:2000) or goat anti-chicken Alexa-Fluor $488(1: 2000)$.

BrdU injections and IHC. To label proliferating cells, two months old mice (three months old in case of GLAST-Dagla and controls, to ensure the full penetrance of the deletion after the tamoxifen injection done at 2 months of age were injected intraperitoneally with 5 -bromo-2'-deoxyuridine (BrdU; $50 \mathrm{mg} / \mathrm{kg}$ body weight, dissolved in sterile saline) once a day for three consecutive days. To investigate proliferation of progenitor cells, 4 mice of each group were killed $24 \mathrm{~h}$ after the last BrdU injection. To determine differentiation and survival of progenitor cells, animals were killed after 21 days. For this, they were anesthetized with isoflurane and transcardially perfused with cooled PBS, following $4 \%$ PFA. Brains were postfixed in $4 \%$ PFA for $24 \mathrm{~h}$ at $4{ }^{\circ} \mathrm{C}$. Brains were cryoprotected in $20 \%$ sucrose for three days at $4{ }^{\circ} \mathrm{C}$ before they were frozen in dry ice cooled isopentane and stored at $-80^{\circ} \mathrm{C}$. Subsequently the entire hippocampus (Bregma -0.94 to $-3.34 \mathrm{~mm}$ ) was cut into 48 serial coronal free-floating cryosections of $40 \mu \mathrm{M}$ thickness. Slices were stored in 48 well plates filled with cryoprotectant (50\% PBS, $25 \%$ glycerol, $25 \%$ ethylenglycol, $0.025 \%$ sodiumazide) at $-20{ }^{\circ} \mathrm{C}$. For immunohistochemical staining, every eighth hippocampal slice of each mouse was selected and transferred in one well of a 24 -wellplate containing Netwell ${ }^{\text {tm }}$ inserts (Costar). All following steps of the staining were done at room temperature under shaking, if not specified otherwise. First, slices were washed three times in TBS for five min to remove remaining cryoprotectant, followed by incubation in permeabilization buffer (TBS containing $0.3 \%$ Tween) for $10 \mathrm{~min}$. After another washing step, slices were incubated in $2 \mathrm{xSSC}$ for $20 \mathrm{~min}$ at $65^{\circ} \mathrm{C}$ for antigen retrieval and then shortly washed in $\mathrm{ddH}_{2} \mathrm{O}$. Since $\mathrm{BrdU}$ is located in the DNA, slices were incubated in $2 \mathrm{M} \mathrm{HCl}$ for $30 \mathrm{~min}$ at $37^{\circ} \mathrm{C}$ to make DNA accessible for the antibody. To neutralize $\mathrm{pH}$, slices were incubated in borate buffer for $10 \mathrm{~min}$ before they were washed in TBS again. Subsequently, slices were blocked in TBS plus II (TBS containing $0.3 \%$ Triton X-100, $5 \%$ goat serum, $2 \%$ BSA) for $1 \mathrm{~h}$.

To investigate the proliferation of progenitor cells, brain slices were incubated with rat anti-BrdU antibody (1:500 ab6326, Abcam in TBS plus II) overnight at $4{ }^{\circ} \mathrm{C}$ under shaking. To investigate the survival and differentiation of progenitor cells, brain slices of mice were also incubated with a mouse NeuN (1:500, conjugated with AF488 MAB377X, Merck) and a rabbit GFAP antibody (1:1000 ab7260, Abcam). Since GFAP is also expressed in young neurons, we also used a S100beta antibody (1:2000 ab41548, Abcam) instead of the GFAP antibody. In both cases, brain slices were incubated in the antibody solutions over night at $4{ }^{\circ} \mathrm{C}$ under shaking.

On the next day, slices were washed and then blocked in TBS plus II for $1 \mathrm{~h}$. As secondary antibody, goat-antirat AF594 (1:500 in TBS plus II) was used to label BrdU antibodies and goat-anti-rabbit AF647 (1:1000 in TBS plus II) to label GFAP- or S100beta-antibodies. Slices were incubated in the secondary antibody solution for $2 \mathrm{~h}$ at RT. After another washing step slices were transferred to coverslips and embedded in DAPI Fluoromount-G ${ }^{\circ}$ (Southern Biotechnology Associates, Inc.). Images of the dentate gyrus region were obtained with Leica SP8 Confocal microscope with $40 \times$ objective in tile-scan mode.

To estimate the rate of neurogenesis, all BrdU-positive cells were counted in the hippocampus from one hemisphere by an experimenter blinded to the genotype of the animals. The following calculation was used:

$$
\mathrm{N}=\Sigma(\text { BrdU cells per animal }) \times\left(\frac{1}{s s f}\right) \times\left(\frac{1}{a s f}\right) \times\left(\frac{1}{t s f}\right) \times 2
$$

The selected sampling fraction (ssf) is 0.125 (6/48), because 6 out of 48 hippocampal slices were analyzed. Since the entire dentate gyrus was used as counting frame, the value for the area sampling fraction (asf) equals 1. Due to the thickness of slices $(40 \mu \mathrm{m})$, the optical section of confocal microscope $(1.3 \mu \mathrm{m})$ and an average diameter of nuclei of $\sim 8 \mu \mathrm{m}$, the thickness of sampling fraction (tsf) was considered as $0.25((1.3 \times 8) / 40)$. Last, the calculated number of BrdU-positive cells was multiplied with two, because only one hemisphere was imaged for analysis.

Since the staining for survival and differentiation (D21) was always performed two times (with GFAP or with s100ß) on 6 slices, the average amount of BrdU-positive and NeuN-positive cells per mouse of both stainings was used for analysis. Differentiation was evaluated by determination of overlaps of BrdU signal and either neuronal- (NeuN) or astrocytic (GFAP/S100ß) markers.

Received: 27 May 2021; Accepted: 22 November 2021

Published online: 12 January 2022

\section{References}

1. Altman, J. \& Das, G. D. Autoradiographic and histological evidence of postnatal hippocampal neurogenesis in rats. J. Comp. Neurol. 124, 319-335 (1965).

2. Bond, A. M., Ming, G. L. \& Song, H. Adult mammalian neural stem cells and neurogenesis: five decades later. Cell Stem Cell 17, 385-395 (2015).

3. Encinas, J. M. et al. Division-coupled astrocytic differentiation and age-related depletion of neural stem cells in the adult hippocampus. Cell Stem Cell 8, 566-579 (2011). 
4. Takei, Y. Age-dependent decline in neurogenesis of the hippocampus and extracellular nucleotides. Hum. Cell 32, 88-94 (2019).

5. Kempermann, G. et al. Human adult neurogenesis: Evidence and remaining questions. Cell Stem Cell 23, 25-30 (2018).

6. Moreno-Jiménez, E. P. et al. Adult hippocampal neurogenesis is abundant in neurologically healthy subjects and drops sharply in patients with Alzheimer's disease. Nat. Med. 25, 554-560 (2019).

7. Aguado, T. et al. The endocannabinoid system drives neural progenitor proliferation. FASEB J. 19, 1704-1706 (2005).

8. Gao, Y. et al. Loss of retrograde endocannabinoid signaling and reduced adult neurogenesis in diacylglycerol lipase knock-out mice. J. Neurosci. 30, 2017-2024 (2010).

9. Jenniches, I. et al. Anxiety, stress, and fear response in mice with reduced endocannabinoid levels. Biol. Psychiatry 79, 858-868 (2016).

10. Piyanova, A. et al. Age-related changes in the endocannabinoid system in the mouse hippocampus. Mech. Ageing Dev. 150, 55-64 (2015).

11. Bilkei-Gorzo, A. The endocannabinoid system in normal and pathological brain ageing. Philos. Trans. R Soc. B Biol. Sci. 367, 3326-3341 (2012).

12. Zimmermann, T. et al. Neural stem cell lineage-specific cannabinoid type-1 receptor regulates neurogenesis and plasticity in the adult mouse hippocampus. Cereb. Cortex 28, 4454-4471 (2018).

13. Schuele, L. L. et al. Diacylglycerol lipase alpha in astrocytes is involved in maternal care and affective behaviors. Glia 69, 377-391 (2021).

14. Mori, T. et al. Inducible gene deletion in astroglia and radial glia-A valuable tool for functional and lineage analysis. Glia 54, 21-34 (2006).

15. Yoshida, T. et al. Localization of diacylglycerol lipase- $\alpha$ around postsynaptic spine suggests close proximity between production site of an endocannabinoid, 2-arachidonoyl-glycerol, and presynaptic cannabinoid CB1 receptor. J. Neurosci. 26, 4740-4751 (2006).

16. Shohayeb, B., Diab, M., Ahmed, M. \& Ng, D. C. H. Factors that influence adult neurogenesis as potential therapy. Transl. Neurodegen. 7, 1-9 (2018).

17. Baptista, P. \& Andrade, J. P. Adult hippocampal neurogenesis: Regulation and possible functional and clinical correlates. Front. Neuroanat. 12, 44 (2018).

18. Dubreucq, S., Koehl, M., Abrous, D. N., Marsicano, G. \& Chaouloff, F. CB1 receptor deficiency decreases wheel-running activity: Consequences on emotional behaviours and hippocampal neurogenesis. Exp. Neurol. 224, 106-113 (2010).

19. Freund, T. F., Katona, I. \& Piomelli, D. Role of endogenous cannabinoids in synaptic signaling. Physiol. Rev. 83, 1017-1066 (2003).

20. Kano, M., Ohno-Shosaku, T., Hashimotodani, Y., Uchigashima, M. \& Watanabe, M. Endocannabinoid-mediated control of synaptic transmission. Physiol. Rev. 89, 309-380 (2009).

21. Maccarrone, M., Guzmán, M., MacKie, K., Doherty, P. \& Harkany, T. Programming of neural cells by (endo)cannabinoids: From physiological rules to emerging therapies. Nat. Rev. Neurosci. 15, 786-801 (2014).

22. Prenderville, J. A., Kelly, Á. M. \& Downer, E. J. The role of cannabinoids in adult neurogenesis. Br. J. Pharmacol. 172, 3950-3963 (2015).

23. Oudin, M. J., Hobbs, C. \& Doherty, P. DAGL-dependent endocannabinoid signalling: Roles in axonal pathfinding, synaptic plasticity and adult neurogenesis. Eur. J. Neurosci. 34, 1634-1646 (2011).

24. Williams, E. J., Walsh, F. S. \& Doherty, P. The FGF receptor uses the endocannabinoid signaling system to couple to an axonal growth response. J. Cell Biol. 160, 481-486 (2003).

25. Galve-Roperh, I. et al. Cannabinoid receptor signaling in progenitor/stem cell proliferation and differentiation. Prog. Lipid Res. 52, 633-650 (2013).

26. Gomez, O. et al. A basal tone of 2-arachidonoylglycerol contributes to early oligodendrocyte progenitor proliferation by activating phosphatidylinositol 3-kinase (PI3K)/AKT and the mammalian target of rapamycin (MTOR) pathways. J. Neuroimmune Pharmacol. 10, 309-317 (2015).

27. Goncalves, M. B. et al. A diacylglycerol lipase-CB2 cannabinoid pathway regulates adult subventricular zone neurogenesis in an age-dependent manner. Mol. Cell. Neurosci. 38, 526-536 (2008).

28. Mensching, L., Djogo, N., Keller, C., Rading, S. \& Karsak, M. Stable adult hippocampal neurogenesis in cannabinoid receptor CB2 deficient mice. Int. J. Mol. Sci. 20, 3759 (2019).

29. Avraham, H. K. et al. Impaired neurogenesis by HIV-1-Gp120 is rescued by genetic deletion of fatty acid amide hydrolase enzyme. Br. J. Pharmacol. 172, 4603-4614 (2015).

30. Bilkei-Gorzo, A. et al. A chronic low dose of $\Delta 9$-tetrahydrocannabinol (THC) restores cognitive function in old mice. Nat. Med. 23, 782-787 (2017).

31. Anacker, C. \& Hen, R. Adult hippocampal neurogenesis and cognitive flexibility-linking memory and mood. Nat. Rev. Neurosci. 18, 335-346 (2017).

32. Du Preez, A. et al. Chronic stress followed by social isolation promotes depressive-like behaviour, alters microglial and astrocyte biology and reduces hippocampal neurogenesis in male mice. Brain. Behav. Immun. 91, 24-47 (2021).

33. Franklin, K. B. J. \& Paxinos, G. The Mouse Brain in Stereotaxic Coordinates. (Boston, 2008).

\section{Acknowledgements}

This research leading to these results was funded by the Deutsche Forschungsgemeinschaft (DFG, German Research Foundation) - project number 324087152, to ABG - project number 426320013 as well as to AZ under Germany's Excellence Strategy - EXC2151 - 390873048, and to EL under the BONFOR Program, Medical Faculty, University of Bonn - O-178.00.0018. We thank our lab members Joanna Komorowska-Müller, Alessandra Gargano, Eva Drews, Edda Erxlebe, Hanna Schrage, Kersin Nicolai, Anne Zimmer, and previous lab members Eva Beins and Imke Jenniches for their help and constructive discussions.

\section{Author contributions}

L.L.S, A.Z., A.B.G. and E.L. were involved in designing research studies. L.L.S., B.S., A.B.G., S.G. and E.L. conducted the experiments, acquired and the analyzed data. A.Z. provided the reagents. L.L.S., A.Z., and E.L. wrote the manuscript. L.L.S., B.S., and E.L. prepared the figures.

\section{Funding}

Open Access funding enabled and organized by Projekt DEAL.

\section{Competing interests}

The authors declare no competing interests. 


\section{Additional information}

Supplementary Information The online version contains supplementary material available at https://doi.org/ 10.1038/s41598-021-04600-1.

Correspondence and requests for materials should be addressed to A.Z.

Reprints and permissions information is available at www.nature.com/reprints.

Publisher's note Springer Nature remains neutral with regard to jurisdictional claims in published maps and institutional affiliations.

(c) (1) Open Access This article is licensed under a Creative Commons Attribution 4.0 International cc) License, which permits use, sharing, adaptation, distribution and reproduction in any medium or format, as long as you give appropriate credit to the original author(s) and the source, provide a link to the Creative Commons licence, and indicate if changes were made. The images or other third party material in this article are included in the article's Creative Commons licence, unless indicated otherwise in a credit line to the material. If material is not included in the article's Creative Commons licence and your intended use is not permitted by statutory regulation or exceeds the permitted use, you will need to obtain permission directly from the copyright holder. To view a copy of this licence, visit http://creativecommons.org/licenses/by/4.0/.

(C) The Author(s) 2022 